\title{
Zero-cycles on projective varieties and the norm principle
}

\author{
Philippe Gille, Nikita Semenov*
}

\begin{abstract}
Using the Gille-Merkurjev norm principle we compute in a uniform way the image of the degree map for quadrics (Springer's theorem), for twisted forms of maximal orthogonal Grassmannians (theorem of Bayer-Fluckiger and Lenstra), for $\mathrm{E}_{6^{-}}$(Rost's theorem), and $\mathrm{E}_{7}$-varieties.
\end{abstract}

Keywords: norm principle, algebraic groups, zero-cycles.

\section{Introduction}

Let $G$ be a simple algebraic group over a field $k$ and $X$ a projective $G$ homogeneous variety. Consider the degree map

$$
\operatorname{deg}: \mathrm{CH}_{0}(X) \rightarrow \mathbb{Z} \text {. }
$$

The goal of the present paper is to provide a method to compute the image of this map (see [PSZ08] for the computation of its kernel).

This problem has a long history starting probably with the Springer theorem which says that an anisotropic quadratic form remains anisotropic over odd degree field extensions. This statement is equivalent to the fact that the image of the degree map when $X$ is an anisotropic quadric equals $2 \mathbb{Z}$.

To stress the difficulty of the above problem note that a computation of the degree map for the varieties of Borel subgroups of groups of type $\mathrm{E}_{8}$ implies Serre's Conjecture II for fields of cohomological dimension $\leq 2$ [Gi97.

\footnotetext{
*Supported partially by DFG, project GI706/1-1.
} 
Moreover, the above problem generalizes Serre's question, whether the map

$$
\mathrm{H}^{1}\left(k, G_{0}\right) \rightarrow \prod \mathrm{H}^{1}\left(K_{i}, G_{0}\right)
$$

has trivial kernel when $K_{i}$ are finite field extensions of $k$ such that $\operatorname{gcd}\left[K_{i}: k\right]=1$ and $G_{0}$ is a split group.

The image of the degree map is known in the following cases: $X$ is a quadric (Springer's theorem), $X$ is a twisted form of a maximal orthogonal Grassmannian (theorem of Bayer-Fluckiger and Lenstra [BFL90]), $X$ is the variety of Borel subgroups of a group of type $\mathrm{F}_{4}, \mathrm{E}_{6}$ (a theorem of Rost, where cohomological invariants of Albert algebras are used), and $\mathrm{E}_{7}$ (Gille's theorem [Gi97], where the norm principle is used). Note also that there are numerous papers of M. Florence, R. Parimala, B. Totaro, and many others concerning closely related problems.

In the present paper we apply the Gille-Merkurjev norm principle [Gi97, [Me96], BM02] and give a uniform proof of the above results. Apart from this, we compute the image of the degree map for the varieties of parabolic subgroups of type 7 of groups $G$ of type $\mathrm{E}_{7}$ and prove that anisotropic groups of type $\mathrm{E}_{7}$ remain anisotropic over odd degree field extensions. Note that this property is used in [PS07, Corollary 6.10] to relate the Rost invariant of $G$ and its isotropity.

\section{Norm principle and strategy of the proof}

2.1. Let $k$ be a perfect field with $\operatorname{char} k \neq 2,3, \Gamma=\operatorname{Gal}(\bar{k} / k)$ the absolute Galois group, $G$ a connected reductive algebraic group over $k, G^{\prime}=[G, G]$ the commutator subgroup, $\Delta$ its Dynkin diagram, and $\Delta_{0}$ its Tits index (see [Ti65]).

2.2 (Special cocharacters). Let $G_{1}$ be a reductive algebraic group over $k$ and

$$
1 \rightarrow G_{1} \rightarrow G \stackrel{f}{\rightarrow} T=\mathbb{G}_{m} \rightarrow 1
$$

an exact sequence. The cocharacter group $T_{*}$ can be canonically identified with the group $\mathbb{Z}$. A cocharacter $\varphi \in T_{*}$ is called $f$-special, if there is a $k$-homomorphism $g: \mathbb{G}_{m} \rightarrow G$ such that $f \circ g=\varphi$.

2.3 (Set $X(\varphi)$ ). Denote $Z^{\prime}=G / G^{\prime}, C$ the center of the simply connected cover of $G^{\prime}, Z$ the center of $G$, and $\mu$ the center of $G^{\prime}$. 
We can represent the homomorphism $f$ as a composition $G \rightarrow Z^{\prime} \rightarrow T$. In particular, there is the induced homomorphism $\alpha: Z^{\prime \Gamma} \rightarrow T_{*}$ between the cocharacter groups. The exact sequence

$$
1 \rightarrow \mu \rightarrow Z \rightarrow Z^{\prime} \rightarrow 1
$$

induces a homomorphism $\beta: Z^{\prime \Gamma}{ }_{*} \rightarrow \mu(-1)^{\Gamma}$, and the canonical epimorphism $C \rightarrow \mu$ induces a map $\gamma: C_{*}^{\Gamma} \rightarrow \mu(-1)^{\Gamma}$, where $\mu(-1)$ is the Tate twist, i.e., $\mu(-1)=\operatorname{Hom}\left(\mu_{n}, \mu\right)$ for any $n$ with $\mu^{n}=1$. For a cocharacter $\varphi \in T_{*}$ we define a subset $X(\varphi) \subset C_{*}^{\Gamma}$ as the set $\gamma^{-1}\left(\beta\left(\alpha^{-1}(\{\varphi\})\right)\right)$.

2.4 (Set $\Omega(\varphi)$ ). From now on we assume that the Dynkin diagram $\Delta$ has no multiple edges. Following [Me96, (5.8)] we identify $C_{*}$ and the character group $C^{*}$ and consider $X(\varphi)$ as a subset of $C^{*}$. Let $\bar{\omega}_{i}$ denote the $i$-th fundamental weight of the simply connected cover of $G^{\prime}$ (Enumeration of simple roots follows Bourbaki). Define now $\Omega(\varphi)$ as the set of all subsets $\Theta \subset \Delta$ such that the elements $\left\{\left.\sum_{i \in I} \bar{\omega}_{i}\right|_{C}, I \subset \Delta \backslash \Theta\right.$ a $\Gamma$-orbit $\}$ generate a subgroup of $C^{* \Gamma}$ that intersects $X(\varphi)$.

2.5 (Type of a parabolic subgroup). It is well-known that there is a bijective correspondence between the conjugancy classes of parabolic subgroups of $G_{\bar{k}}^{\prime}$ and the subsets of the set $\Delta$ of simple roots.

The type of a parabolic subgroup is the corresponding subset of $\Delta$. Under this identification the Borel subgroup has type $\emptyset$. If $P$ is a maximal parabolic subgroup of type $\Delta \backslash\left\{\alpha_{i}\right\}$, where $\alpha_{i}$ is the $i$-th simple root, then for simplicity of notation we say that $P$ is of type $i$.

2.6 (Tits homomorphism). Let

$$
\beta: C^{* \Gamma} \rightarrow \operatorname{Br}(k)
$$

be the Tits homomorphism for the simply connected cover of $G^{\prime}$ defined in [Ti71]. In order to compute the sets $\Omega(\varphi)$ we need to know the restrictions of the fundamental weights $\bar{\omega}_{i}$ to $C$ and their images under the Tits homomorphism.

Below we describe them for groups of type ${ }^{1} \mathrm{D}_{n}, \mathrm{E}_{6}$, and $\mathrm{E}_{7}$. We use graphical notation, where the algebra over a vertex $i$ of the Dynkin diagram stands for the image $\beta\left(\left.\bar{\omega}_{i}\right|_{C}\right)$. Apart from this, the restriction $\left.\bar{\omega}_{i}\right|_{C}$ is trivial iff the respective algebra is $k$. 
Type $\mathrm{D}_{n}$ : A simply connected group of inner type $\mathrm{D}_{n}$ has the form $\operatorname{Spin}(A, \sigma)$, where $A$ is a central simple algebra of degree $2 n$ with an orthogonal involution $\sigma$ of the first kind with trivial discriminant.

For the character group $C^{*}$ of the center of $\operatorname{Spin}(A, \sigma)$ we have

$$
C^{*}=\left\{0, \chi, \chi^{+}, \chi^{-}\right\}
$$

where $\chi$ (resp. $\chi^{+}, \chi^{-}$) is the restriction of the fundamental weight $\bar{\omega}_{1}$ (resp. $\left.\bar{\omega}_{n-1}, \bar{\omega}_{n}\right)$ to the center.

Let $C^{ \pm}(A, \sigma)$ be the direct summands of the Clifford algebra $C_{0}(A, \sigma)=$ $C^{+}(A, \sigma) \oplus C^{-}(A, \sigma)$. We have

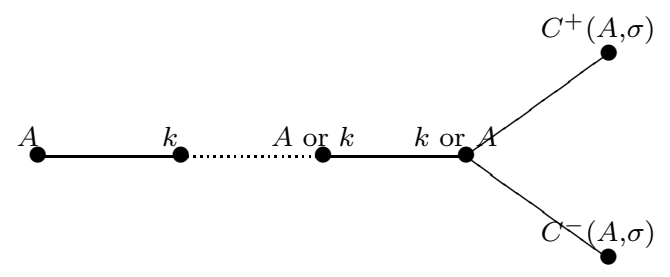

We associate the Tits algebras to the last two vertices $n-1$ and $n$ in such a way that for $\varepsilon=+$ (resp. $\varepsilon=-)$ the algebra $C^{\varepsilon}(A, \sigma)$ splits over the field of rational functions of the projective homogeneous variety of maximal parabolic subgroups of type $P_{n-1}\left(\right.$ resp. $\left.P_{n}\right)$. The latter are two irreducible components of the variety of $2 n^{2}$-dimensional isotropic right ideals $I$ of $A$ with respect to $\sigma$.

Type $\mathrm{E}_{6}$ : The Tits algebra is a certain central simple algebra $A$ of index 1 , 3,9 , or 27 and of exponent 1 or 3 .

Type $\mathrm{E}_{7}$ : The Tits algebra is a certain central simple algebra $A$ of index 1 , 2,4 , or 8 and of exponent 1 or 2 .

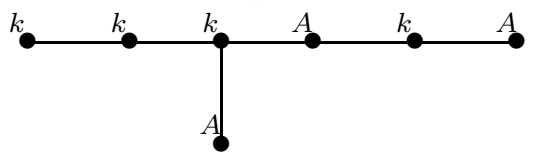

Under the above assumptions the following lemmas hold:

2.7 Lemma ([Me96, Lemma 3.4]). Let $K / k$ be a finite field extension lying in the algebraic closure $\bar{k}$ and let $\varphi \in T_{*}$. If the cocharacter $\varphi$ is $f_{K}$-special, then the cocharacter $[K: k] \varphi$ is $f$-special. 
2.8 Lemma ([Me96, Theorem 5.6]). For a cocharacter $\varphi \in T_{*}$ the following conditions are equivalent:

1. $\varphi$ is $f$-special;

2. there exists a parabolic subgroup of $G$ defined over $k$ whose type is contained in $\Omega(\varphi)$.

2.9 Lemma ([Me96, Proposition 5.8]). Let $\beta: C^{* \Gamma} \rightarrow \operatorname{Br}(k)$ be the Tits homomorphism for the simply connected cover of $G^{\prime}$. Assume that the Dynkin diagram $\Delta$ has no multiple edges. If a cocharacter $\varphi \in T_{*}$ is $f$-special, then $0 \in \beta(X(\varphi))$.

2.10 Theorem. Let $X$ be an anisotropic smooth projective variety over $k$ and $p$ a prime number. In the above notation assume that the following conditions hold:

1. For any field extension $K / k$ and for any coprime to $p$ cocharacter $\varphi$, if $0 \in \beta_{K}(X(\varphi)) \subset \operatorname{Br}(K)$ and $G^{\prime}$ has a parabolic subgroup defined over $K$ whose type is contained in $\Omega(\varphi)$, then $X(K) \neq \emptyset$;

2. For any field extension $K / k$ and for any coprime to $p$ cocharacter $\varphi$ if $X(K) \neq \emptyset$, then there exists a parabolic subgroup of $G^{\prime}$ of type contained in $\Omega(\varphi)$ defined over $K$.

Then $\operatorname{deg}\left(\mathrm{CH}_{0}(X)\right) \subset p \mathbb{Z}$.

Proof. Let $K / k$ be a field extension. We show first the following

Claim. $X(K) \neq \emptyset$ if and only if any coprime to $p$ cocharacter $\varphi$ is $f_{K}$-special.

Indeed, if $X(K) \neq \emptyset$, then by item 2 there is a parabolic subgroup of $G^{\prime}$ defined over $K$ whose type is contained in $\Omega(\varphi)$. By Lemma $2.8 \varphi$ is $f_{K}$-special.

Conversely, if $\varphi$ is $f_{K^{-}}$special, then by Lemma 2.9 we have $0 \in \beta_{K}(X(\varphi))$, and by Lemma 2.8 there is a parabolic subgroup of $G^{\prime}$ defined over $K$ of type contained in $\Omega(\varphi)$. Therefore by item 1 we have $X(K) \neq \emptyset$.

Let now $K / k$ be a finite field extension such that $X(K) \neq \emptyset$. To finish the proof of the theorem it sufficies to show that $[K: k]$ is divisible by $p$. Assume the converse.

Since $X(K) \neq \emptyset$, by Claim any coprime to $p$ cocharacter $\varphi$ is $f_{K^{-}}$special. By Lemma 2.7 the cocharacter $[K: k] \varphi$ is $f$-special. Therefore by Claim $X(k) \neq \emptyset$. Contradiction. 


\section{Applications}

3.1 Corollary (Springer's theorem). Let $A$ be a central simple $k$-algebra of degree $2 n \geq 4$ with an orthogonal involution $\sigma$ of the first kind. Let $X$ be the variety of isotropic with respect to $\sigma$ right ideals of $A$ of dimension $2 n$. Assume $X$ is anisotropic.

Then $\operatorname{deg}\left(\mathrm{CH}_{0}(X)\right) \subset 2 \mathbb{Z}$. In particular, if $X$ is an anisotropic smooth even-dimensional projective quadric, then $\operatorname{deg}\left(\mathrm{CH}_{0}(X)\right)=2 \mathbb{Z}$.

Proof. ([Me96, Lemma 6.2]). There is the following exact sequence of groups:

$$
1 \rightarrow G_{1}=\operatorname{Spin}(A, \sigma) \rightarrow G=\Gamma(A, \sigma) \stackrel{f}{\rightarrow} \mathbb{G}_{m} \rightarrow 1,
$$

where $\Gamma(A, \sigma)$ is the Clifford group and $f$ is the spinor norm homomorphism.

Let $p=2$. It is easy to check that for any odd cocharacter $\varphi$ the set $X(\varphi)=\{\chi\}$, where $\chi$ is the restriction of $\bar{\omega}_{1}$ to the center $C$.

Let $K / k$ be a field extension. If $0 \in \beta_{K}(X(\varphi))$, then the algebra $A_{K}$ is split (see 2.6). Thus, $\sigma_{K}$ corresponds to a quadratic form, and $X_{K}$ is a projective quadric. If additionally $G_{K}^{\prime}$ has a parabolic subgroup defined over $K$ of type contained in $\Omega(\varphi)$, then it easy to see that this quadratic form is isotropic, and thus $X(K) \neq \emptyset$.

Finally, if $X(K) \neq \emptyset$, then $G^{\prime}$ has a parabolic subgroup of type $\Delta \backslash\left\{\alpha_{1}\right\}$, where $\alpha_{1}$ is the first simple root. But $\Delta \backslash\left\{\alpha_{1}\right\} \in \Omega(\varphi)$.

Thus, we checked all conditions of Theorem 2.10.

3.2 Corollary (Bayer-Fluckiger and Lenstra). Let A be a central simple algebra of degree $2 n \geq 4$ with an orthogonal involution $\sigma$ of the first kind. Let $Y$ be the variety of $2 n^{2}$-dimensional isotropic right ideals of $A$ and

$$
X=Y \times \operatorname{Spec}\left(k[t] /\left(t^{2}-\operatorname{disc}(\sigma)\right) .\right.
$$

Assume $X$ is anisotropic. Then $\operatorname{deg}\left(\mathrm{CH}_{0}(X)\right) \subset 2 \mathbb{Z}$.

Proof. ([Me96, 6.3]). Consider the following exact sequence of groups:

$$
1 \rightarrow G_{1}=\mathrm{O}^{+}(A, \sigma) \rightarrow G=\mathrm{GO}^{+}(A, \sigma) \stackrel{f}{\rightarrow} \mathbb{G}_{m} \rightarrow 1,
$$

where $f$ is the multiplier map. 
Let $p=2$. Denote as $\chi^{+}$(resp. $\chi^{-}$) the restriction of the fundamental weight $\bar{\omega}_{n-1}$ (resp. $\left.\bar{\omega}_{n}\right)$ to the center. It is easy to check that for any odd $\operatorname{cocharacter} \varphi$ we have

$$
X(\varphi)= \begin{cases}\emptyset, & \operatorname{disc}(\sigma) \neq 1 \\ \left\{\chi^{+}, \chi^{-}\right\}, & \operatorname{disc}(\sigma)=1 .\end{cases}
$$

Finally, if $0 \in \beta_{K}(X(\varphi))$, then $\operatorname{disc}\left(\sigma_{K}\right)=1$. Then the variety $Y_{K}$ is the disjoint union of varieties of parabolic subgroups of $G_{K}^{\prime}$ of types $\Delta \backslash\left\{\alpha_{n-1}\right\}$ and $\Delta \backslash\left\{\alpha_{n}\right\}$. If additinally $G^{\prime}$ has a parabolic subgroup defined over $K$ of type contained in $\Omega(\varphi)$, then the Tits index $\Delta_{0}$ of $G_{K}^{\prime}$ contains at most one of the roots $\alpha_{n-1}, \alpha_{n}$ (see 2.6). Therefore in this case $X(K) \neq \emptyset$.

To finish the proof of the corollary it remains to notice that condition 2 of Theorem 2.10 is obvious.

Using similar arguments one can show the following well-known statement. Opposite to the traditional approach our proof does not use cohomological invariants of Albert algebras.

3.3 Corollary (M. Rost). Let $G_{1}$ be a simply connected algebraic group of type ${ }^{1} \mathrm{E}_{6}$ over $k$ and $X$ the variety of its parabolic subgroups of type 1 (resp. 6). Assume $X$ is anisotropic. Then $\mathrm{CH}_{0}(X) \subset 3 \mathbb{Z}$.

Proof. If $G_{1}$ has a non-trivial Tits algebra, then the statement is obvious, since for a field extension $K / k$ condition $X(K) \neq \emptyset$ implies that the Tits algebras of $\left(G_{1}\right)_{K}$ are split.

Assume that $G_{1}$ has trivial Tits algebras. Let $J$ be an Albert algebra associated with $G_{1}$ (see Ga01a]). A $k$-linear map

$$
h: J \rightarrow J
$$

is called a similarity if there exists $\alpha_{h} \in k^{\times}$(the multiplier of $h$ ) such that

$$
N(h(j))=\alpha_{h} N(j)
$$

for all $j \in J$, where $N$ stands for the cubic norm on $J$. Then $G_{1}$ coincides with the similarities of this Jordan algebra with multiplier 1 . Let $G$ be the group of all similarities. Then $G$ is a reductive group and there is the following exact sequence of algebraic groups:

$$
1 \rightarrow G_{1} \rightarrow G \stackrel{f}{\rightarrow} T=\mathbb{G}_{m} \rightarrow 1
$$


where the map $f$ is defined on $k$-points as $h \mapsto \alpha_{h}$.

Let $p=3$ and let $\varphi \in T_{*}=\mathbb{Z}$ be a cocharacter coprime to 3 . We check now the conditions of Theorem 2.10 .

First we compute $X(\varphi)$. In our situation $G^{\prime}=[G, G]=G_{1}, Z^{\prime}=T=$ $\mathbb{G}_{m}, \mu=\mu_{3}, C=\mu_{3}, Z=\mathbb{G}_{m}$, and the group

$$
C_{*} \simeq C^{*}=\mathbb{Z} / 3=\left\{0,\left.\bar{\omega}_{1}\right|_{C},\left.\bar{\omega}_{6}\right|_{C}=-\left.\bar{\omega}_{1}\right|_{C}\right\}
$$

where $\left.\bar{\omega}_{i}\right|_{C}$ denotes the restriction of the $i$-th fundamental weight of $G_{1}$ to the center, $i=1,6$. Therefore, $X(\varphi)=\left\{\left.\bar{\omega}_{1}\right|_{C}\right\}$ or $X(\varphi)=\left\{\left.\bar{\omega}_{6}\right|_{C}\right\}$ (it depends on $\varphi \bmod 3)$.

Let $K / k$ be a field extension. Assume first that $G_{K}^{\prime}$ is isotropic and the type of a parabolic subgroup $P$ of $G^{\prime}$ defined over $K$ is contained in $\Omega(\varphi)$. If the parabolic subgroup of $G^{\prime}$ of type 1 is not defined, then by Tits's classification [Ti65, p. 58] the Tits index of $G^{\prime}$ equals $\Delta_{0}=\Delta \backslash\left\{\alpha_{2}, \alpha_{4}\right\}$. But the restrictions to the center of the 2 -nd and of the 4 -th fundamental weights are trivial (see [Ti90, p. 653] or 2.6). This contradicts to the assumption that the type of $P$ is contained in $\Omega(\varphi)$.

Finally, condition 2 of Theorem 2.10 is obvious.

3.4 Remark. If the Tits algebras of $G_{1}$ are trivial, then the image of the degree homomorphism $\mathrm{CH}_{0}(X) \rightarrow \mathbb{Z}$ equals $3 \mathbb{Z}$.

3.5 Corollary. Let $G_{1}$ be a simply connected algebraic group of type $\mathrm{E}_{7}$ over $k$ and $X$ the variety of maximal parabolic subgroup of $G_{1}$ of type 7 . Assume $X$ is anisotropic. Then $\mathrm{CH}_{0}(X) \subset 2 \mathbb{Z}$.

Proof. Let $(A, \sigma, \pi)$, where $A$ is a central simple $k$-algebra with a symplectic involution $\sigma$ and $\pi: A \rightarrow A$ a linear map, be a gift associated with $G_{1}$ (see [Fe72], Ga01a] and [Ga01b]). An invertible element $h \in A$ is called a similarity if there exists $\alpha_{h} \in k^{\times}$(the multiplier of $h$ ) such that

$$
\sigma(h) h=\alpha_{h} \cdot 1
$$

and

$$
\pi\left(h a h^{-1}\right)=\alpha_{h} h \pi(a) h^{-1}
$$

for all $a \in A$. Then $G_{1}$ coincides with the similarities of this gift with multiplier 1. Let $G$ be the group of all similarities. Then $G$ is a connected reductive group and there is the following exact sequence of algebraic groups:

$$
1 \rightarrow G_{1} \rightarrow G \stackrel{f}{\rightarrow} T=\mathbb{G}_{m} \rightarrow 1,
$$


where the map $f$ is defined on $k$-points as $h \mapsto \alpha_{h}$.

Let $p=2$ and let $\varphi$ be an odd cocharacter.

First we compute $X(\varphi)$. In our situation $G^{\prime}=[G, G]=G_{1}, Z^{\prime}=T=$ $\mathbb{G}_{m}, \mu=\mu_{2}, C=\mu_{2}$, and $Z=\mathbb{G}_{m}$. Thus, the maps $\alpha$ and $\gamma$ from 2.3 are the identity maps. The map $\beta: Z_{*}^{\prime}=\mathbb{Z} \rightarrow \mu_{2}(-1)=\mathbb{Z} / 2$ from 2.3 is the usual factor-map. Therefore, $X(\varphi)=\{\chi\}$, where as $\chi$ we denote a unique non-trivial element of $C_{*} \simeq C^{*}$.

Let $K / k$ be a field extension. Assume that $0 \in \beta_{K}(X(\varphi)), G_{K}^{\prime}$ is isotropic and the type of a parabolic subgroup of $G^{\prime}$ defined over $K$ is contained in $\Omega(\varphi)$. The first assumption implies that the Tits algebra $A$ of $G_{1}$ is split.

If the parabolic subgroup of $G^{\prime}$ of type 7 is not defined, then by Tits's classification [Ti65, p. 59] the Tits index of $G^{\prime}$ equals $\Delta_{0}=\Delta \backslash\left\{\alpha_{1}\right\}$. But the

restriction to the center of the 1-st fundamental weight is trivial (see [Ti90, p. 653]). Therefore we have $X(K) \neq \emptyset$.

Finally, condition 2 of Theorem 2.10 is obvious.

3.6 Remark. If the Tits algebras of $G_{1}$ are trivial, then the image of the degree homomophism $\mathrm{CH}_{0}(X) \rightarrow \mathbb{Z}$ equals $2 \mathbb{Z}$.

3.7 Corollary. A group $G_{1}$ as in the statement of the previous corollary does not split over an odd degree field extension.

\section{References}

[BM02] P. Barquero, A. Merkurjev. Norm Principle for Reductive Algebraic Groups, Algebra, arithmetic and geometry, Part I, II (Mumbai, 2000), 123-137, Tata Inst. Fund. Res. Stud. Math. 16 (2002), Bombay.

[BFL90] E. Bayer-Fluckiger, H. W., Jr. Lenstra. Forms in odd degree extensions and self-dual normal bases. Amer. J. Math. 112 (1990), 359-373.

[Fe72] J.C. Ferrar. Strictly regular elements in Freudenthal triple systems. Trans. Amer. Math. Soc., 174 (1972), 313-331.

[Ga01a] S. Garibaldi. Structurable algebras and groups of type $\mathrm{E}_{6}$ and $\mathrm{E}_{7}$. J. Algebra 236 (2001), no. 2, 651-691. 
[Ga01b] S. Garibaldi. Groups of type $\mathrm{E}_{7}$ over arbitrary fields. Comm. Alg. 29 (2001), no. 6, 2689-2710.

[Gi97] $\mathrm{Ph}$. Gille. La $R$-equivalence sur les groupes algébriques réductifs définis sur un corps global. Inst. Hautes Études Sci. Publ. Math. 86 (1997), 199-235.

[Gi97] Ph. Gille. Cohomologie galoisienne des groupes quasi-déployés sur des corps de dimension cohomologique $\leq 2$, Compositio Math. 125 (2001), 283-325.

[Me96] A. Merkurjev. A norm principle for algebraic groups. St. Petersburg Math. J. 7 (1996), no. 2, 243-264.

[PS07] V. Petrov, N. Semenov. Higher Tits indices of algebraic groups. Appendix by M. Florence. 2007. Available from http://arxiv.org/abs/0706.2827

[PSZ08] V. Petrov, N. Semenov, K. Zainoulline. Zero-cycles on a Cayley plane. Canad. Math. Bull. 51 (2008), no. 1, 114-124.

[Ti65] J. Tits. Classification of algebraic semisimple groups. In Algebraic Groups and Discontinuous Subgroups (Proc. Sympos. Pure Math., Boulder, Colo., 1965), 33-62. Amer. Math. Soc., Providence, R.I., 1966.

[Ti71] J. Tits. Représentations linéaires irréductibles d'un groupe réductif sur un corps quelconque. J. reine angew. Math. 247 (1971), 196-220.

[Ti90] J. Tits. Strongly inner anisotropic forms of simple algebraic groups. J. Algebra 131 (1990), no. 2, 648-677.

UMR 8553 du CNRS, École Normale Supérieure, 45 Rue D’Ulm, 75005 PARIS, France

Mathematisches Institut, Universität München, Theresienstr. 39, 80333 München, Germany 